Dans un dernier volet (mais pas le moindre) sont présentées les activités entreprises par diverses organisations. Le FISE/UNICEF tout d'abord, par son directeur exécutif adjoint, le $\operatorname{Dr} C$. A. Egger, un de nos compatriotes, et la Direction de la coopération au développement $\mathrm{du}$ gouvernement suisse (Wilhelm et Zanolli). Puis six organisations privées, cinq dont l'objectif premier est l'action pratique dans le cadre de projets de développement, Frères des Hommes (Mongin), Helvetas (Leisinger et Gerster), Medicus Mundi (Widmer), Swissaid (Schnellmann), et une orientée principalement vers la recherche, la Fondation Nestlé (Aebi et coll.).

Tout cela permet-il de dire que tout va pour le mieux dans la coopération technique issue de Suisse? Il serait un peu présomptueux de le prétendre. Entre autres choses parce que quantitativement la contribution de notre pays reste certainement insuffisante. Sur le plan qualitatif, l'auteur de cet éditorial a la témérité de penser que, dans l'ensemble, les communications de nos collègues donnent une impression saine; que les remises en question, les approches et les actions pratiques actuelles vont largement dans le bon sens. Sur cette base, on peut espérer que ce fascicule de Médecine sociale et préventive apportera sa pierre à l'effort d'information et de sensibilisation si nécessaire dans les pays industrialisés en ce qui concerne une véritable action conjointe (dans le même sens!) avec ceux avec qui, quoi qu'on fasse, nous sommes dans la même nacelle (le «Spaceship Earth»).

Les connaissances, les compétences et les attitudes à disposition apparaissent honorables. Manquent des ressources. Serons-nous assez convaincants pour en obtenir plus, de façon que l'effort de la Suisse se rapproche de ceux relativement bien plus importants d'autres petits pays industrialisés, de l'Europe du Nord notamment (avec lesquels par ailleurs nous avons passablement de points communs). En janvier dernier, le Conseiller fédéral Aubert, dans un exposé fait à Zurich devant un institut de recherches internationales, soulignait le rôle marquant que peuvent jouer les petits pays, sur le plan diplomatique par exemple, et même sur le plan militaire. Il est tout à fait imaginable que, dans les années qui viennent et si la collectivité accepte d'y mettre le prix, la Suisse joue un rôle nettement plus substantiel que par le passé dans le domaine de la coopération.

Pour que, dans l'avenir, le diagnostic qu'on peut poser sur la coopération au développement soit plus proche de la «bonne santé», de la pleine santé.

\title{
Editorial
}

\section{Entwicklungszusammenarbeit im Gesundheitswesen: krank, rekonvaleszent oder gesund?}

\section{J. Martin ${ }^{1}$}

Das vorliegende Heft von «Sozial- und Präventivmedizin» stellt mehr Fragen, als es Antworten gibt. Nachdem die Vereinigten Nationen ein Jahrzehnt der Entwicklung ausgerufen hatten, befinden wir uns jetzt am Ende des zweiten, und die Beurteilung des zurückgelegten Weges bleibt ziemlich unklar und oft nicht sehr optimistisch. Man spricht von «Stagflation» in den industrialisierten Ländern und von «Wachstum ohne Entwicklung» in der Dritten Welt; oft gibt es ernsthafte Gründe für die Annahme, dass sich in den benachteiligten Ländern das Los der Mehrheit der Bevölkerung verschlechtert.

1 Dr méd., médecin cantonal adjoint, Service de la santé publique, $\mathrm{CH}-1001$ Lausanne.
Die Gegner der Entwicklungshilfe und die Skeptiker schlagen uns vor, der Devise Hugos $\mathrm{zu}$ folgen, «unsere Kuh zu melken und in Frieden zu leben». Auf einer anderen Ebene der Analyse beschreiben Illich und seine Gruppe von Cuernavaca die Irrwege des aufdringlichen, auf alles übergreifenden westlichen Entwicklungsmodells und rufen nach einer Gesellschaftsform des friedlichen $\mathrm{Zu}$ sammenlebens. In diesem Zusammenhang wird man mit Interesse die Bemerkungen Benjamin Franklins über «die Wilden Nordamerikas» wieder lesen. Haben wir seither in konzeptueller Hinsicht Fortschritte gemacht?

Alle diejenigen, welche der Ansicht sind, dass etwas unternommen werden soll, betonen aber, dass die 
Aufhebung der technischen Hilfe die zunehmenden unerwünschten Auswirkungen der industriellen Zivilisation in der Dritten Welt nicht verhindern wird. Dies wird beispielsweise von den Autoren des Artikels über die Entwicklungsarbeit in Bhutan einem lange Zeit von der übrigen Welt isolierten Land - zum Ausdruck gebracht. Es ist bekannt, dass der Tourismus Devisen, aber auch eine ganze Reihe von Úbeln, wovon die Prostitution eines der sichtbarsten ist, mit sich bringt. In den Massenmedien der Entwicklungsländer herrscht in der Regel ein niedriges Niveau kosmopolitischen Denkens, und erzielte Fortschritte sind durch Änderungen in den politischen Orientierungen bedroht usw.

Aber nichts ist absolut richtig oder absolut falsch. Der Schweizer entdeckt im fremden Lande, dass gewisse, ja viele Probleme nicht wirklich gut gelöst werden können; es gibt nur mehr oder weniger schlechte Lösungen (im Gegensatz zu dem, was man bei uns zu glauben scheint). Man soll dann die besten unter den letzteren suchen. In diesem Rahmen ist es nicht unnütz, seinen Beitrag zu leisten und sich aktiv einzusetzen; das haben die Autoren und die Organisationen, welche an diesem Heft mitarbeiten, beschlossen.

Eine der Hauptlinien dieses Heftes ist ohne Zweifel die Art und Weise, in der so viele Individuen und Gruppen die Notwendigkeit einer Veränderung beschreiben. Die Notwendigkeit, im Laufe eines schwierigen Prozesses eine neue Konzeption ihrer Rolle zu erarbeiten, eine Konzeption, die in erster Linie der Realität und nicht lobenswerten Gefühlen oder einer Moral Rechnung trägt. Man kehrt nicht von einem Unternehmen der Entwicklungszusammenarbeit zurück, ohne dass man sich gezwungen fühlt, seine Ansichten und Prioritäten grundlegend neu zu gestalten. Man kann sogar sagen, dass es sich in dieser Einsicht um eine ohne Zweifel positive Folge der Anstrengungen in der technischen Zusammenarbeit der letzten Jahrzehnte handelt und dass diese Einsicht dem eigenen Lande zugute kommen kann. Ein ernst zu nehmendes Problem besteht jedoch darin, dass es sich in diesem Falle um einen Nutzen für den "Geber» handelt; es wird aber unablässig betont, dass der Nutzen der Entwicklungshilfe in erster Linie und in vollem Umfange den Entwicklungsländern zugute kommen muss. Müssen wir daran erinnern, dass die Befunde in dieser Hinsicht jedoch oft ambivalent sind?

Damit die Anstrengungen der Zusammenarbeit in den Partnerländern nennenswerte Früchte zeitigen, ist es unbedingt erforderlich, dass man sich einige Regeln zu eigen macht, deren wichtigste wahrscheinlich «Zuhören, zuhören und nochmals zuhören» ist. Über die Regeln und Techniken hinaus spielen sowohl auf der einen als auch auf der ande- ren Seite die Einstellungen eine entscheidende Rolle. An einer Zusammenkunft in Genf beharrte Professor Lambo, ein Staatsangehöriger Nigerias und heute Stellvertretender Generaldirektor der WHO, in der technischen Zusammenarbeit auf einer «brutalen Offenheit» und darauf, dass diese eine bis dahin unveränderbare Höflichkeit in den meisten Situationen zu ersetzen habe.

Die in dieser Nummer vereinigten Mitteilungen stellen einen vielseitigen und für die von unserem Lande in der Entwicklungszusammenarbeit im Gesundheitswesen einzunehmende Haltung hinreichend beweiskräftigen Fächer dar. Einige der hier vorgestellten Organisationen verfügen bereits über eine langjährige Geschichte ihrer Arbeit in der Dritten Welt, andere sind jünger; einige sind öffentlich, andere privat. Einige Artikel beschreiben, wie sich die Dinge in den traditionellen Strukturen abspielen, ohne Intervention von aussen. Viele legen das Hauptgewicht auf die Notwendigkeit der Erziehung der Massen, auf die Teilnahme der Bevölkerung, die primäre Gesundheitsversorgung und die Fachkräfte in der primären Gesundheitsversorgung («primary health workers»).

Kann man aufgrund dieser Artikel sagen, dass in der schweizerischen technischen Zusammenarbeit alles zum besten bestellt ist? Dies zu behaupten wäre überheblich, unter anderem auch deshalb, weil der Beitrag unseres Landes in quantitativer Hinsicht sicherlich ungenügend ist. In qualitativer Hinsicht und aus der Sicht dieser Ausgabe scheint es, dass sich die vorhandenen Kenntnisse, Kompetenzen und Haltungen durchaus sehen lassen können. Was fehlt, sind die Mittel. Werden wir genügend überzeugen können, um mehr zu erreichen, so dass die Anstrengungen der Schweiz sich den vergleichsweise viel grösseren Anstrengungen anderer kleiner industrialisierter Länder hauptsächlich Nordeuropas annähern?

Im vergangenen Januar unterstrich Bundesrat Aubert in einem Vortrag in Zürich die wichtige Rolle, welche die kleinen Länder zum Beispiel auf diplomatischer Ebene und sogar in militärischer Hinsicht spielen können. Es ist durchaus denkbar, dass die Schweiz auf dem Gebiet der Zusammenarbeit in den folgenden Jahren, und wenn die Gesellschaft bereit ist, den Preis zu bezahlen, eine gewichtigere Rolle spielen wird als in der Vergangenheit.

Schliesslich bleibt zu wünschen, dass diese Nummer der «Sozial- und Präventivmedizin» ihren Baustein beiträgt zu den unerlässlichen Bemühungen in der Information und, wenn notwendig, in der Sensibilisierung der industrialisierten Länder, im Hinblick auf eine mit den Entwicklungsländern gemeinsam unternommene Aktion mit vereinten Kräften. 